\title{
Dynamic Control of Location-Specific Information in Tactile Cutaneous Reflexes from the Foot during Human Walking
}

\author{
Bart M. H. Van Wezel, Frans A. M. Ottenhoff, and Jacques Duysens \\ Department of Medical Physics and Biophysics, University of Nijmegen, 6525 EZ Nijmegen, The Netherlands
}

The purpose of the present study was to determine whether tactile cutaneous reflexes from the skin of the foot contain location-specific information during human walking. Muscular responses to non-nociceptive electrical stimulation of the sural, posterior tibial, and superficial peroneal nerves, each supplying a different skin area of the foot, were studied in both legs during walking on a treadmill. For all three nerves the major responses in all muscles were observed at a similar latency of $\sim 80-85$ msec. In the ipsilateral leg these reflex responses and their phase-dependent modulation were highly nerve-specific. During most of the stance phase, for example, the peroneal and tibial nerves generally evoked small responses in the biceps femoris muscle. In contrast, during late swing large facilitations generally occurred for the peroneal nerve, whereas suppressions were observed for the tibial nerve. In the contralateral leg the reflex responses for the three nerves were less distinct, although some nerve specificity was observed for individual subjects. It is concluded that non-nociceptive stimulation of the sural, posterior tibial, and superficial peroneal nerves each evokes distinct reflex responses, indicating the presence of location-specific information from the skin of the foot in cutaneous reflexes during human walking. It will be argued that differentially controlled reflex pathways can account for the differences in the phase-dependent reflex modulation patterns of the three nerves, which points to the dynamic control of this information during the course of a step cycle.

Key words: cutaneous reflexes; phase-dependent reflex modulation; local sign; location-specific information in reflexes; human walking; sural nerve; superficial peroneal nerve; posterior tibial nerve; EMG
The various skin areas of the foot seem well suited to provide specific tactile information about events that are encountered during everyday human locomotion. Reflexes are thought to contribute in the processing of such information because an electrical stimulus at a tactile, non-nociceptive intensity applied at cutaneous nerves supplying the skin of the foot evokes responses at $\sim 80$ msec, which are specifically controlled according to the phase of the step cycle, the muscle, and the leg in which they occur (Duysens et al., 1990, 1991, 1996; Yang and Stein, 1990; De Serres et al., 1995; Tax et al., 1995). So far, only the effects of individual nerves supplying a restricted skin area at the foot have been studied. However, information about the location of a given event (i.e., spatial information) may be essential to provide for an appropriate reaction. For example, mechanical taps to different sides of the foot elicit different responses during cat locomotion (Buford and Smith, 1993).

In static conditions, reflex responses in leg muscles after cutaneous stimulation of the limb depend on the location of the stimulus ("local sign") in the human (Hagbarth, 1960; Kugelberg et al., 1960; Meinck et al., 1981; Aniss et al., 1992), in the cat

Received Dec. 5, 1996; revised Feb. 13, 1997; accepted Feb. 21, 1997.

This work was supported by a North Atlantic Treaty Organization Grant to J.D. (CRG 910574), by a Grant from the European Community (ESPRIT BRA 6615), by the Foundation for Life Sciences, by the Netherlands Organization for Scientific Research, and by the Prinses Beatrix Fonds. We thank L. H. P. Murrer for his participation in the experiments. We also thank G. Windau for software development, A. M. van Dreumel and J. W. C. Kleijnen for their technical assistance, and Dr

A. A. M. Tax and A. M. Schillings for their critical reading of an early version of this manuscript.

Correspondence should be addressed to Bart M. H. Van Wezel, Department of Medical Physics and Biophysics, University of Nijmegen, Geert Grooteplein 21, 6525 EZ Nijmegen, The Netherlands.

Dr. Ottenhoff's present address: Bakken Research Center, Endepolsdomein 5, 6229 GW Maastricht, The Netherlands.

Copyright (C) 1997 Society for Neuroscience $0270-6474 / 97 / 173804-11 \$ 05.00 / 0$
(Sherrington, 1910; Hagbarth, 1952; Engberg, 1964; Hongo et al., 1990; LaBella and McCrea, 1990; Leahy and Durkovic, 1991), and in invertebrates such as the locust (Siegler and Burrows, 1986). This indicates the presence of segregations in the reflex pathways of the various cutaneous nerves, which can be used to provide location-specific information from the skin of the foot during locomotion. In fact, nerve-specific reflex effects to purely cutaneous stimulation have been observed during cat locomotion (Abraham et al., 1985; Moschovakis et al., 1991; Pratt et al., 1991; LaBella et al., 1992; Degtyarenko et al., 1996), indicating the presence of location-specific information. For example, in the intact walking cat inhibitory responses in the semitendinosus were found only to sural nerve stimulation and not to stimulation of other cutaneous nerves (Pratt et al., 1991). Still, an overall common synergy of flexor responses in the swing phase and extensor responses in the stance phase was observed, independent of the location of the stimulus (Duysens and Stein, 1978; Duysens and Loeb, 1980; Abraham et al., 1985) (for review, see Rossignol et al., 1988). Hence, both common and nerve-specific control of cutaneous reflex responses were observed during cat locomotion.

It is unknown whether tactile cutaneous reflexes from the skin of the foot contain location-specific information during human locomotion. Therefore, the present study focused on the responses to selective non-nociceptive stimulation of the sural, posterior tibial, and the superficial peroneal nerves. Each of these three nerves contains cutaneous afferents from its own specific innervation area at the foot. Responses were studied in biceps femoris (BF) and semitendinosus (ST), because the ipsilateral sural nerve elicits a specific reflex synergy of large facilitatory responses in BF and smaller or even suppressive responses in ST during human locomotion (Tax et al., 1995; Duysens et al., 1996). This synergy could be related exclusively to the innervation area of 
the sural nerve (lateral side of the foot), because BF and ST have antagonistic tasks with respect to foot exo/endorotation (Tax et al., 1995). Possible concomitant nerve-specific responses also were studied in their antagonist rectus femoris. Responses were studied in tibialis anterior as well, because a reversal from facilitatory responses during early swing to suppressive responses during end swing for both posterior tibial and sural nerve stimulation was observed in this muscle (Yang and Stein, 1990; De Serres et al., 1995; Tax et al., 1995; Duysens et al., 1996), which could point to stereotyped reflex responses during human locomotion. The same muscles of the other leg were measured also to investigate whether there exist contralateral nerve-specific reflexes.

Preliminary results have been published in abstract form (Van Wezel et al., 1994).

\section{MATERIALS AND METHODS}

The methods in this study were mostly identical to those that have been described in detail elsewhere (Tax et al., 1995; Duysens et al., 1996). The essentials will be described below, together with some specific procedures.

Experimental set-up. Experiments were performed on a group of 10 normal healthy subjects ( 8 male, 2 female) aged between 19 and 46 . The experiments were performed in conformity with the declaration of Helsinki for experiments on humans. All subjects had given informed consent and had no known history of neurological or motor disorder. They were asked to walk on a treadmill at $4 \mathrm{~km} / \mathrm{hr}$ while wearing a safety harness that was fastened to an emergency brake at the ceiling.

A bipolar stimulation electrode (with poles of $0.5 \mathrm{~cm}$ and an inter-pole distance of $2.0 \mathrm{~cm}$ ) was positioned over the left sural (approximately halfway between the lateral malleolus and the Achilles tendon), posterior tibial (approximately halfway between the medial malleolus and the Achilles tendon), or superficial peroneal (on the proximal dorsal side of the foot) nerve, firmly pressed with elastic straps. For each subject the exact position of the stimulation electrode was determined according to the optimal irradiation of the stimulus, corresponding to the innervation area of the nerve involved. The electrical stimulus consisted of a train of five rectangular pulses of $1 \mathrm{msec}$ duration given at $200 \mathrm{~Hz}$. A custom-made constant-current stimulator provided the desired stimulus amplitude.

Very thin insole foot-switch systems (designed in collaboration with Algra Fotometaal b.v., Wormerveer, The Netherlands) were used to detect foot contact. Bipolar electromyographic (EMG) activity was recorded in both the ipsilateral (stimulated) and contralateral (unstimulated) leg by means of surface electrodes over the long head of the biceps femoris (iBF and $\mathrm{cBF}$ for ipsilateral and contralateral biceps femoris, respectively), the semitendinosus (iST and cST), the rectus femoris (iRF and cRF), and the tibialis anterior (iTA and cTA) muscles. The EMG signals were (pre-) amplified (by a total factor in the order of $10^{6}$ ), high-pass-filtered (cut-off frequency at $3 \mathrm{~Hz}$ ), full-wave-rectified, and then low-pass-filtered (cut-off frequency at $300 \mathrm{~Hz}$ ). In addition, the ipsilateral ankle angle was measured with a laterally placed goniometer to examine possible kinesiological effects of the EMG responses. These signals were sampled along with the stimulus voltage and current (measured with a sample-and-hold circuit), the ipsilateral and contralateral foot-switch signals, and a digital code referring to the stimulus condition. The data were sampled at $500 \mathrm{~Hz}$ and stored on hard disk for each trial

Experimental protocol. The subjects were trained to walk at a comfortable, constant pace with the belt speed set at $4 \mathrm{~km} / \mathrm{hr}$. The experiment on a subject consisted of three consecutive experimental runs in which one of the three nerves (sural, posterior tibial, or superficial peroneal nerve) was stimulated.

Before each experimental run, during quiet standing, the perception threshold (PT) after stimulation of the corresponding nerve was determined by gradually increasing (to above PT) and decreasing (to below PT) the stimulus amplitude. Before the start of an experimental run the PT had to be stable before and after a short walking period. During the experimental runs the intensity of the stimuli was always 2 PT. This intensity was chosen because it gives a tactile, non-nociceptive sensation on the specific area of the foot that is innervated by the stimulated nerve in all subjects. Furthermore, it elicits reflex responses similar to those at even lower intensities (Duysens et al., 1990, 1991, 1996; Tax et al., 1995), suggesting involvement of low-threshold cutaneous afferent fibers.

The stimuli were delivered at 16 preprogrammed and equidistantly distributed points over the whole step cycle. All data were measured starting $100 \mathrm{msec}$ before stimulation and lasting for $1600 \mathrm{msec}$. During the same periods control values (i.e., no stimulus) of the EMG also were measured. Both ipsilateral and contralateral footfall served as reference points for the time in the step cycle, each for one-half of the total step cycle (see Tax et al., 1995). Every stimulus condition was presented 10 times in an experimental run. All 320 trials occurred in random order. The successive stimulus conditions were separated by a random interval in the range of 3.5-6.5 sec. Hence, two stimuli were always separated by at least two step cycles without a stimulus. At the end of each experimental run the perception threshold was determined once more. It was slightly lowered ( $\sim 6 \%$ on average), as compared with the measurement taken immediately before the experimental run.

Stimulus stability. Both voltage and current of the stimulus were quite constant over the 16 points of the step cycle. On average, the maximum deviation from the mean of the 16 points of the step cycle was $<3 \%$ for the voltage and $<1.5 \%$ for the current. However, this does not rule out the possibility that the nerve itself might receive a variable input because of changes in the ankle positions during the course of a step cycle. In previous publications it was discussed that this possibility did not play a large role (Duysens et al., 1995, 1996). Nevertheless, to check this possibility, in one subject the compound sensory action potentials of the sural and peroneal nerves were checked for several ankle positions under stationary conditions. The ankle positions ranged from $70^{\circ}$ (plantar flexion) to $115^{\circ}$ (dorsiflexion), both of which are beyond the range of ankle movements during walking (Inman et al., 1981). In none of the conditions were the compound sensory action potentials significantly different. Furthermore, if there had been a large decrease of stimulus intensity delivered to the nerve at any given point of the step cycle during walking, one would expect a decrease in response in all muscles. This was not observed in any of the subjects (see Results). In addition, the responses of the ipsilateral tibialis anterior muscle in this study agree with those of other groups (see Results), including those that have controlled for the stimulation by using mixed nerve stimulation and monitoring of the M-waves (Yang and Stein, 1990; De Serres et al., 1995). Hence, there are no indications that there is a large phase-dependent variation of the stimulus delivered to the nerves.

Data analysis. The overall effect after nerve stimulation was obtained by averaging the 10 trials of all 32 stimulus conditions (16 stimulus phases with and 16 control phases without stimulation) and subsequently by subtracting the resulting control data from the corresponding stimulus data ("pure" responses or "subtractions"). Hence, for each experiment (i.e., stimulated nerve) and for each muscle 16 subtraction traces were obtained, corresponding to the 16 phases in the step cycle (see Fig. 1). For all three nerves, responses could be observed in all muscles at a latency of $\sim 80 \mathrm{msec}$, consistent with previous literature (Yang and Stein, 1990; Duysens et al., 1991, 1996; Tax et al., 1995). Quantification of the responses occurred by calculating the mean of the EMG data over the period in which the responses occurred. Therefore, for each nerve/muscle combination one single time window was set around the responses for all 16 phases in the step cycle (cf. Yang and Stein, 1990; Duysens et al., 1991, 1996; Tax et al., 1995) (see Fig. 1). When a muscle showed little or no response, no adequate window could be set. In that case an average window was used, calculated from the time windows used to measure responses (in order of priority) in other nearby muscles in the same leg or the same muscle for other nerves (cf. Tax et al., 1995).

For each trial the mean EMG value was calculated within the applicable window obtained from the above analysis. Subsequently, for all conditions (i.e., for 16 phases both control and stimulus conditions), the average and the SE were calculated from these window-averaged trials $(n=10)$. The resulting data underwent an amplitude normalization (with respect to the maximum of the 16 averaged control values) and a time normalization (a subdivision of the step cycle into 16 phases) so that a proper intersubject comparison could be made. To determine whether the observed reflex responses were statistically significant, the responses underwent a Wilcoxon signed rank test (significance level, $p<0.05$ ).

\section{RESULTS}

\section{Timing of the responses}

To obtain the average net effect after stimulation at the nonnociceptive intensity of two times perception threshold (PT), we averaged the 10 trials of each stimulus condition, and we subsequently subtracted the average control trials from the corresponding average stimulus trials. An example is shown in Figure 1 for 

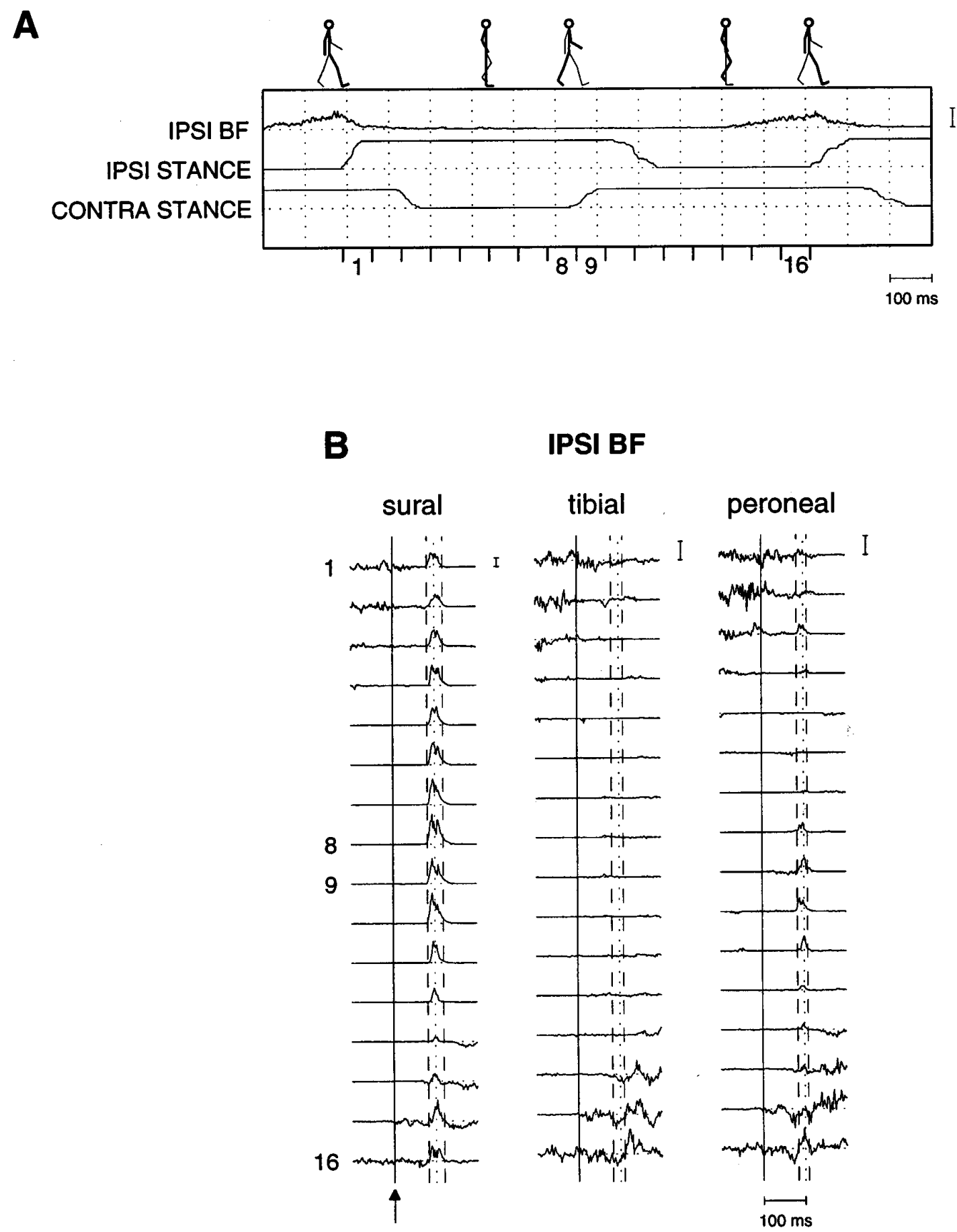

\section{IPSI BF}

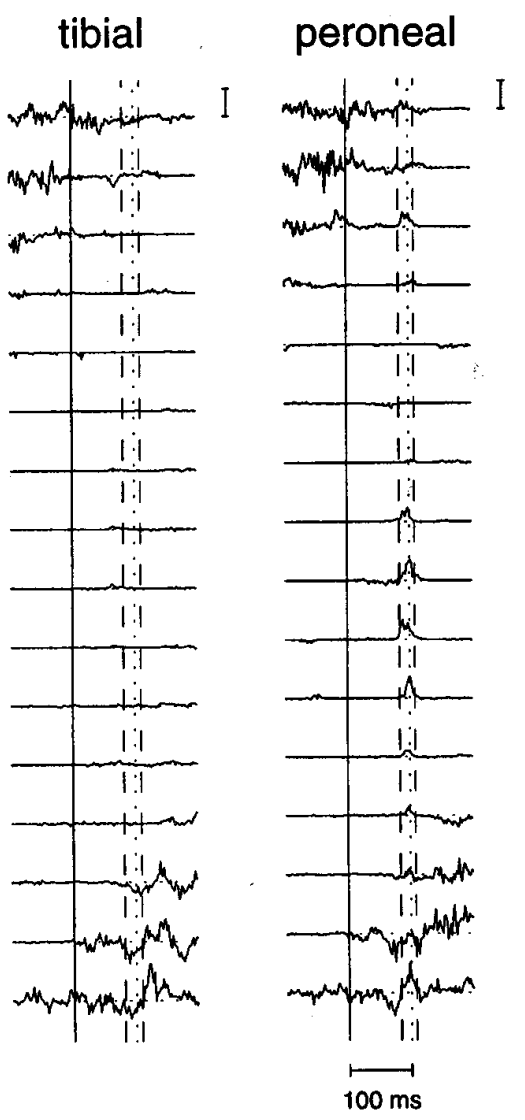

STIMULATION

Figure 1. Ipsilateral biceps femoris (iBF) of subject 1. A, iBF background locomotor activity in relation to the stance (signal up) and swing phases of both legs. Stick diagrams are shown on top. One single step cycle is subdivided into 16 equal intervals, the beginning of the first interval coinciding with ipsilateral footfall (see Materials and Methods). $B$, For the iBF a typical set of 48 average ( $n=10$ trials) subtraction traces is shown. This set is subdivided into three subsets that refer to the three different nerves that were stimulated (sural, posterior tibial, and superficial peroneal nerves). The solid lines indicate the time of stimulation, whereas the dotted lines refer to a delay of $100 \mathrm{msec}$ with respect to the onset of stimulation. The stippled lines indicate the time windows that are set around the responses (see Materials and Methods). The time windows were set, using the responses of only those phases in which the maximum exceeded $1 \mathrm{SD}$ of the corresponding background activity. Because the maximum is the value of one single data point, the subtracted data first were smoothed (with a second-order low-pass digital Butterworth filter having a cut-off frequency of $50 \mathrm{~Hz}$ ) so that a reliable determination of the maximum was possible. This maximum was sought within 50 and $130 \mathrm{msec}$ after the stimulus to assure that the responses with a latency of $\sim 80 \mathrm{msec}$ were certainly within that time scale. It then was checked whether the value of this maximum exceeded 1 SD of the unsmoothed background activity. When this was the case, the subtracted data of that phase were selected for determination of the time window. For this subject the time windows for iBF were $80-110,80-108$, and $82-106$ for the sural, tibial, and peroneal nerves, respectively. The number on the left side of each subtraction trace indicates in which of the 16 step cycle intervals the response with a latency of $\sim 80 \mathrm{msec}$ occurs. In all panels: time calibration, $100 \mathrm{msec}$; EMG calibration, $1 \mathrm{mV}$. Note that for visual purposes the size of the EMG calibration bars differs for the three nerves. 


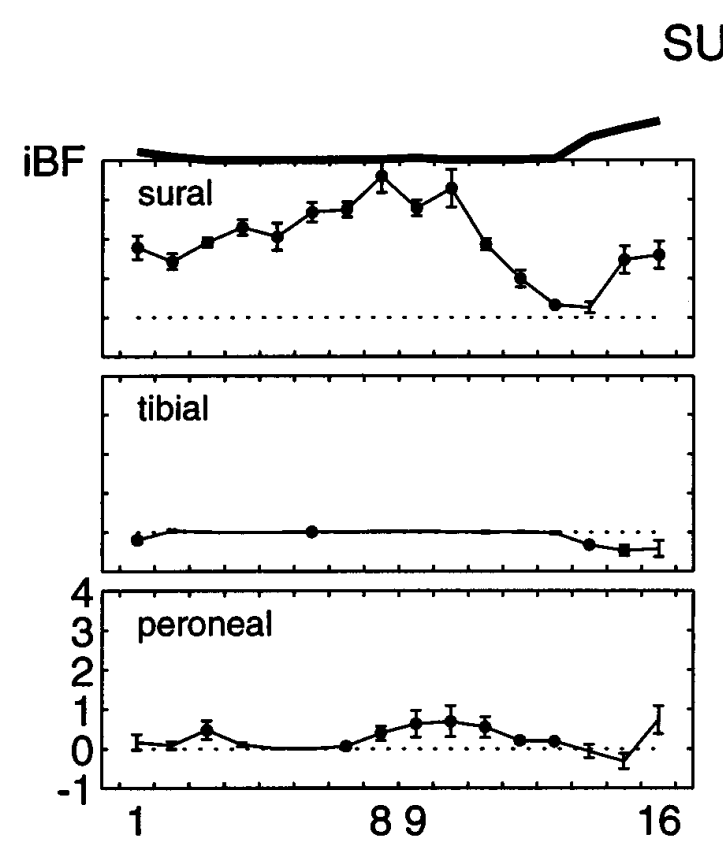

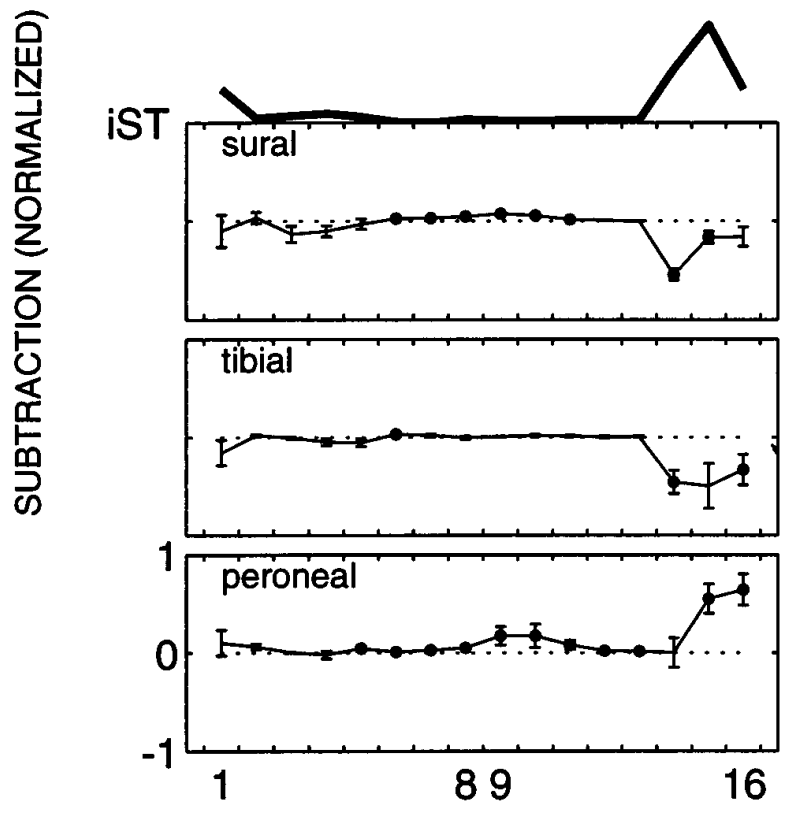

PHASE IN STEP CYCLE

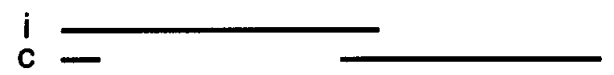

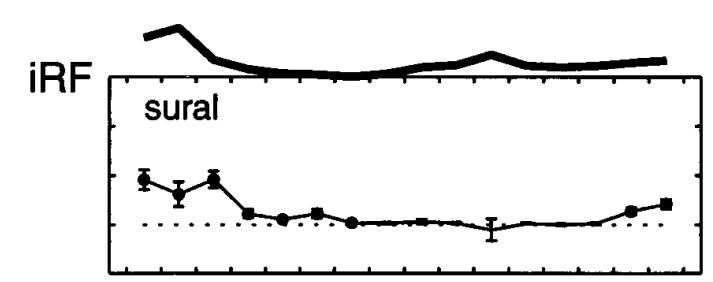
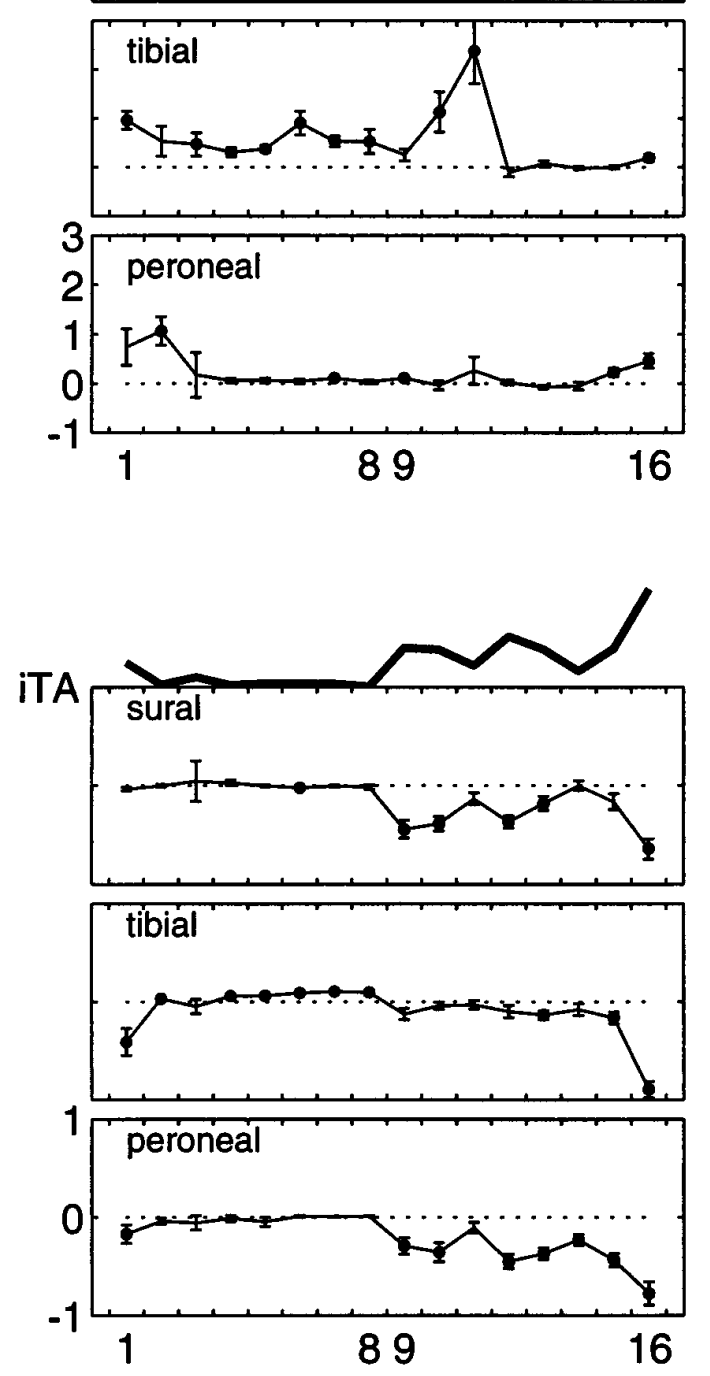

PHASE IN STEP CYCLE

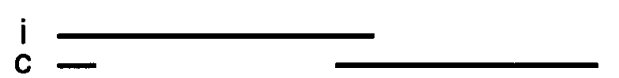

Figure 2. Ipsilateral muscles of subject 1. For the three nerves the subtracted responses ( \pm SE, $n=10$ trials) are plotted for all four ipsilateral muscles as a function of the phase in the step cycle. The statistical significance of the reflexes (Wilcoxon signed rank test, $p<0.05$ ) is indicated by the black dots. Phases 1 and 9 correspond to the onset of ipsilateral and contralateral stance, respectively. On the top of these figures the background activity of the corresponding muscle is shown. The data are normalized with respect to the maximum background locomotor activity of each muscle (see Materials and Methods). The ipsilateral $(i)$ and contralateral $(c)$ stance phases are shown at the bottom. Note the different vertical scales for the different muscles.

the $\mathrm{iBF}$ of subject 1 . For each stimulated nerve 16 subtraction traces are shown corresponding to the 16 phases of stimulation in the step cycle (Fig. $1 B$ ). Reflex responses can be observed with a latency of $\sim 80 \mathrm{msec}$ for all three nerves. The strength and sign of these responses could be different for the different nerves. For example, both sural and peroneal nerve stimulation evoked predominantly facilitatory responses in $\mathrm{iBF}$ for this subject (Fig. 1B). These were clearly largest for the sural nerve and could be larger than the corresponding background activity (Fig. $1 A$ ). In contrast, tibial nerve stimulation evoked predominantly suppressive re- 

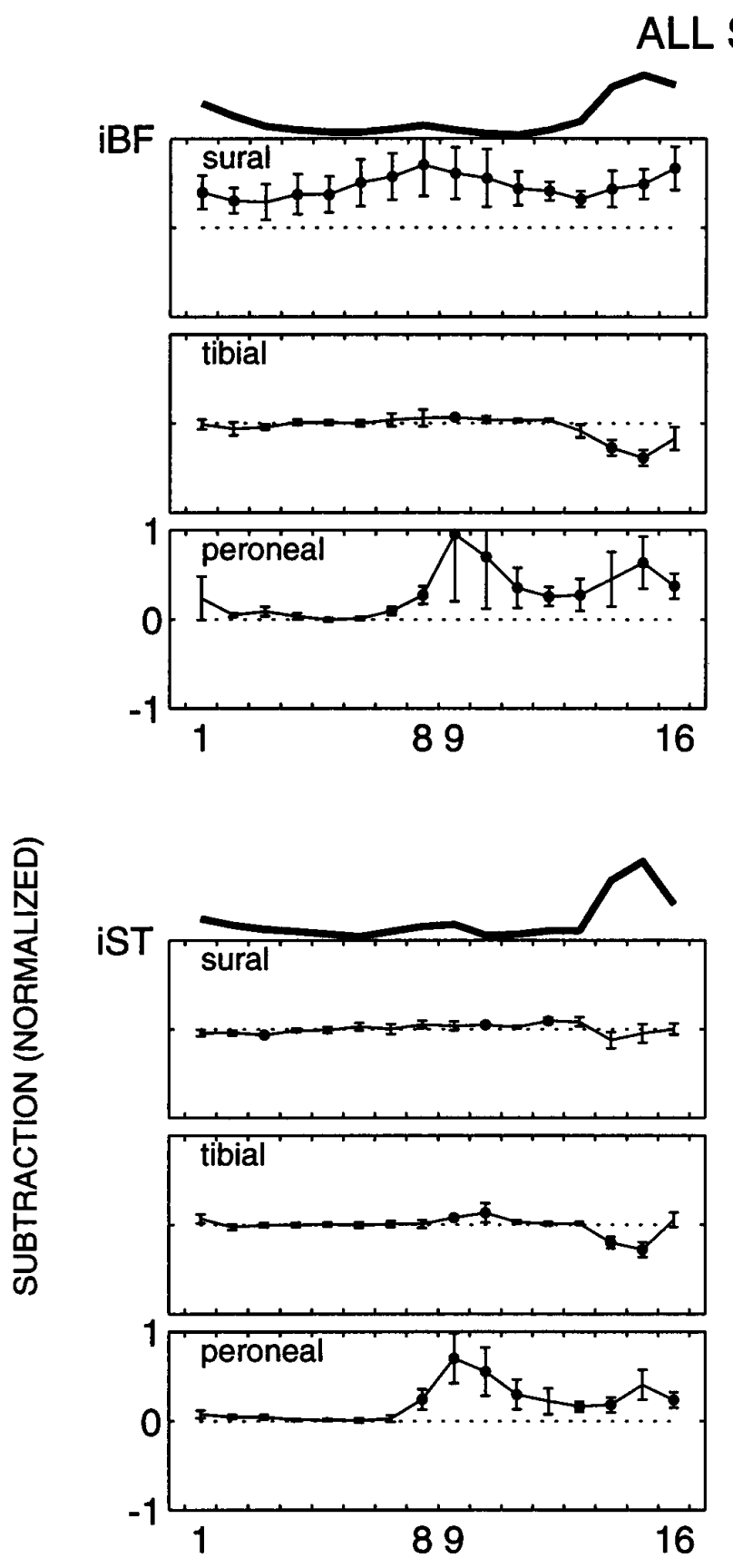

PHASE IN STEP CYCLE

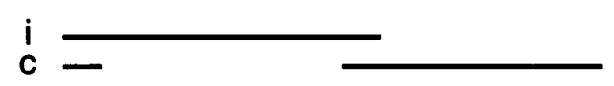

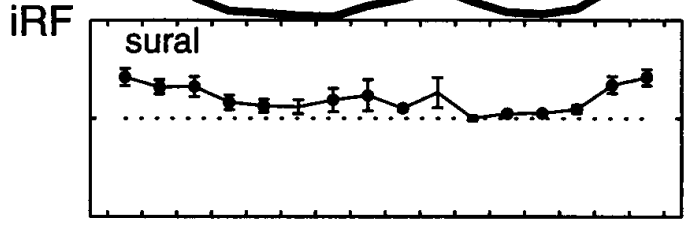
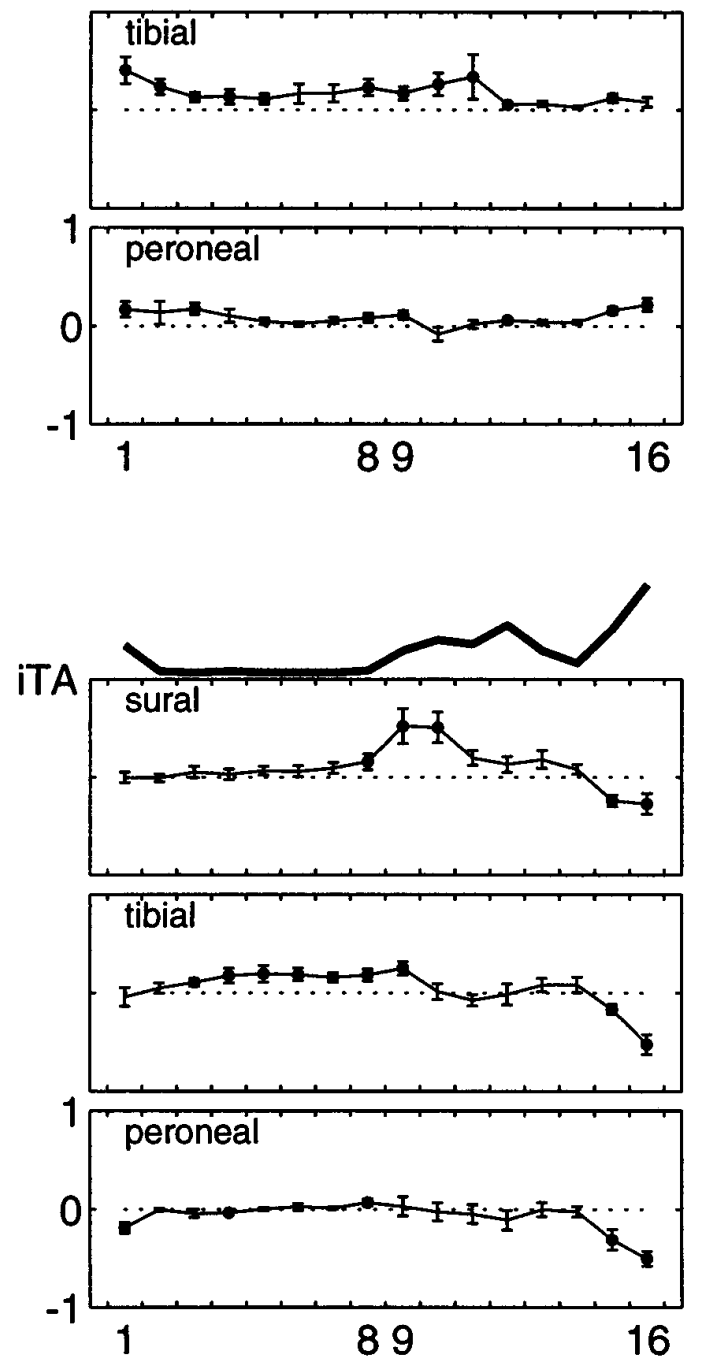

PHASE IN STEP CYCLE

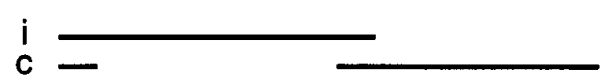

Figure 3. Population average of the ipsilateral muscles $(n=10$ subjects). The same format is used as in Figure 2 .

sponses at this latency (Fig. $1 B$, phases 14-16), which were statistically significant in phases 14 and 15 (see Fig. 2).

The latency of these responses did not exhibit a significant phase-dependent effect, in contrast to the magnitude (Fig. 1B). To quantify the magnitude of the responses, we set a time window around the responses (see Materials and Methods). For all three nerves and in all muscles, responses occurred at comparable latencies of $\sim 80-85 \mathrm{msec}$ (mean of sural, tibial, and peroneal was
85,84 , and $84 \mathrm{msec}$, respectively), with durations of $\sim 30 \mathrm{msec}$ (mean of sural, tibial, and peroneal was 30, 29, and $29 \mathrm{msec}$, respectively) in all muscles, both ipsilateral and contralateral to the side of stimulation. Consistent with previous results (Tax et al., 1995), the latency of the responses in the contralateral leg (mean $86 \mathrm{msec}$ ) was at most a few milliseconds larger than that in the ipsilateral leg (mean $83 \mathrm{msec}$ ). Responses with a latency of $\sim 50 \mathrm{msec}$ occasionally also were observed in the ipsilateral leg, 


\section{IPSILATERAL ANKLE}

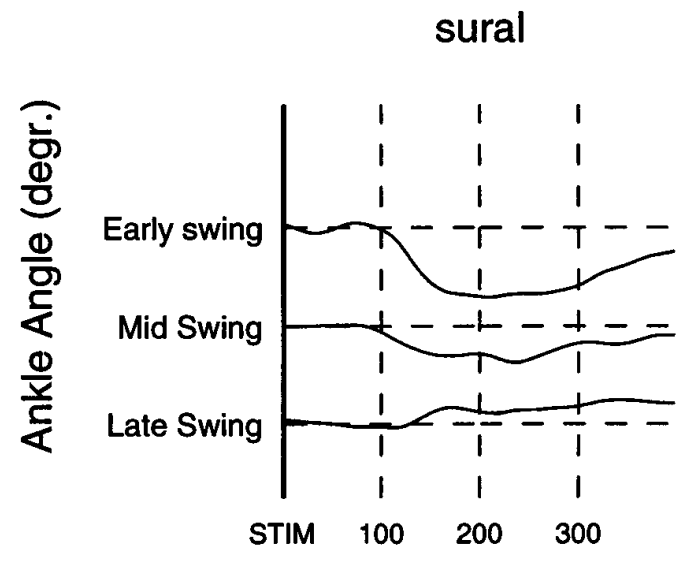

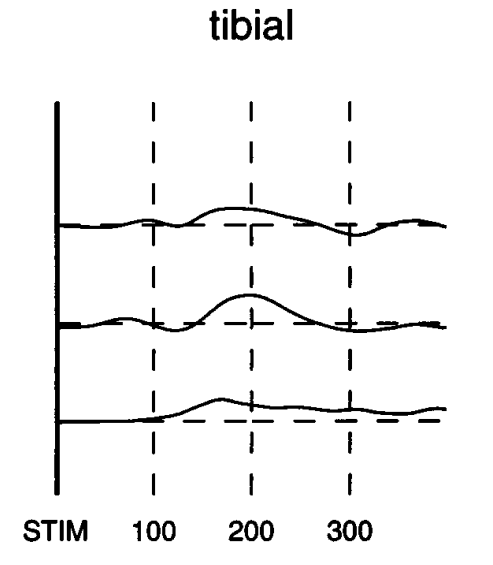

tibial

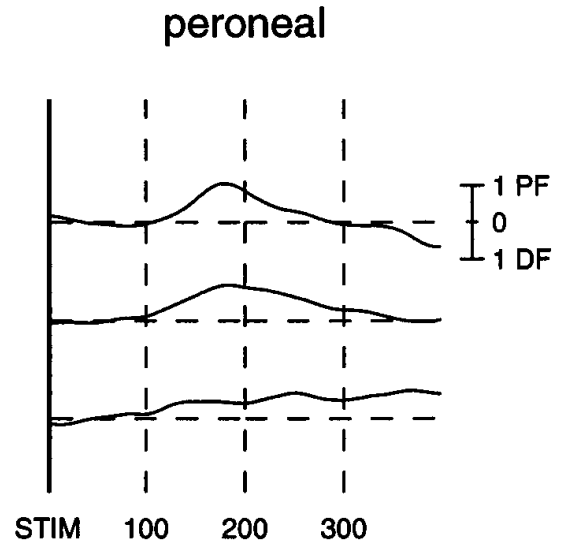

\section{Time after stimulation (ms)}

Figure 4. Subtracted ankle angles during the swing phase. On the basis of the dominant iTA modulation pattern (see Fig. 3), kinesiological data were averaged for the sural ( $n=9$ subjects), tibial $(n=9)$, and peroneal $(n=7)$ nerves. Periods are averages of phases 10-11 (early swing), 12-14 (mid-swing), and 15-16 (late swing). Thick solid lines indicate time of stimulation. The dashed lines are set at 100 msec with respect to each other. Ankle calibration in degrees: $P F$, plantar flexion; $D F$, dorsiflexion.

but these were both small and inconsistent across muscles, nerves, and subjects. Hence, attention will be focused on the responses with a latency of $\sim 80-85 \mathrm{msec}$ (cf. Yang and Stein, 1990; De Serres et al., 1995; Tax et al., 1995; Duysens et al., 1996).

\section{Phase-dependent reflex modulation}

\section{Ipsilateral responses}

For all 320 trials in each experiment, the mean EMG value was calculated within the applicable window obtained from the above analysis (see Materials and Methods). Subsequently, for all stimulus conditions (i.e., for 16 phases, both control and stimulus conditions) both the average and the SE were calculated for these window-averaged trials ( $n=10$ trials per stimulus condition). The results of subject 1 of the ipsilateral responses for all nerves are given in Figure 2. In addition, the population averages are given in Figure 3. For each muscle investigated, averages and SE of the subtracted responses are plotted according to their appearance in the 16 phases of the step cycle. A statistically significant difference (Wilcoxon signed rank test, $p<0.05$ ) between control and reflex data are indicated by black dots. Note that small responses that occur during periods with low or absent background activity often reach a statistically significant level (e.g., Fig. 2, iST to sural nerve stimulation, phases $6-11$ ). This is because low background activities usually exhibit a low background noise level, making it easier for responses to reach significance levels (although small responses tend to be slightly underestimated by the present methods; see Baker and Lemon, 1995). On top of the panels in Figures 2 and 3 , the background activity of the corresponding muscle is shown as well, to facilitate a direct comparison between the responses and the background locomotor pattern.

The reflex responses in $\mathrm{BBF}$ were different for the three nerves both for single subjects (Fig. 2) and for the whole population (Fig. 3). Sural nerve reflex responses were mostly facilitatory over the whole step cycle in this muscle. In contrast, during early stance to mid-swing the tibial nerve reflex responses were small (Fig. 3) or close to zero (Fig. 2), whereas during the second half of swing statistically significant suppressions were observed (Figs. 2, 3, phases 14 and 15). Peroneal nerve stimulation evoked small or no responses throughout most of the stance phase (Figs. 2, 3). At the end of the stance phase facilitatory responses generally were evoked, which persisted throughout the swing phase in most (Fig. 3), but not all (Fig. 2), cases. The observed differences indicate that the modulation of the reflex responses as a function of the phase in the step cycle is different for the three nerves.

The results on the other muscles confirmed this finding. For iST the responses to sural and tibial nerve stimulation were small during stance and the first half of swing. During end swing, suppressions were observed mostly (Fig. 2), which were statistically significant for the whole population for tibial nerve stimulation (Fig. 3). During most of the stance phase, iST responses to peroneal nerve stimulation were small or zero in all subjects, similar to the responses of the other two nerves (Fig. 3). During end stance to end swing, in contrast, the peroneal nerve showed facilitatory responses (Figs. 2, 3). Hence, during some periods in the step cycle the responses could be similar for the nerves, but in other periods they were not.

Note that for both tibial and peroneal nerve stimulation the response synergy of the two hamstring muscles iBF and iST was comparable during the whole step cycle, whereas sural nerve stimulation evoked responses that were larger in iBF than in iST (Wilcoxon signed rank test, $p<0.01$ ). In five subjects sural nerve stimulation even evoked opposite responses in iBF (facilitations) and iST (suppressions) in certain phases of the step cycle (e.g., Fig. 2, phases 14 and 15).

For all three nerves, the responses in iRF approximately followed the background activity, being largest during the stance-toswing and swing-to-stance transitions. Hence, a common response modulation was found in iRF for the three nerves. In eight of the subjects the responses were smallest for peroneal nerve stimula- 

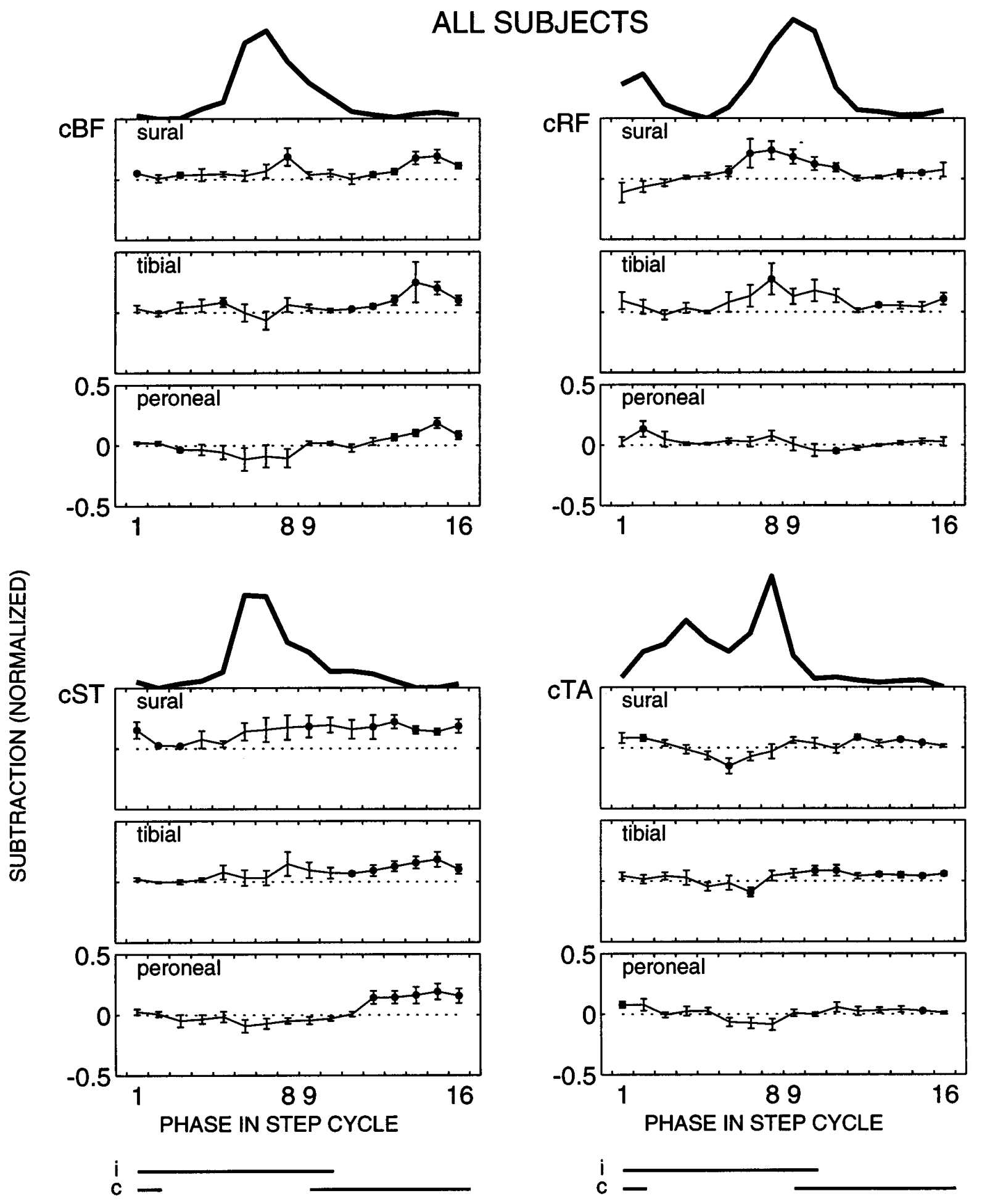

Figure 5. Population average of the contralateral muscles $(n=10$ subjects). The same format is used as in Figure 2.

tion (Fig. 3). For the population the response patterns of the sural and tibial nerve were similar, namely facilitatory during both background activity bursts (Fig. 3). Hence, the nerve-specific response synergy observed in the hamstring muscles usually was not accompanied by a differential synergy in this antagonist. Nevertheless, in some subjects the phase dependency was nervespecific. In subject 1 , for example, the iRF responses during the stance-to-swing transition were significantly larger for the tibial nerve than for those of the sural and peroneal nerves (Fig. 2).

For iTA there were also some striking similarities across nerves. In contrast to $\mathrm{iRF}$, however, these clearly were not related to the background activity. For all three nerves a consistent suppressive response pattern was observed in phases 15 and 16, although the background activity was usually largest during this period (Fig. 3). 


\section{SUBJECT 2}

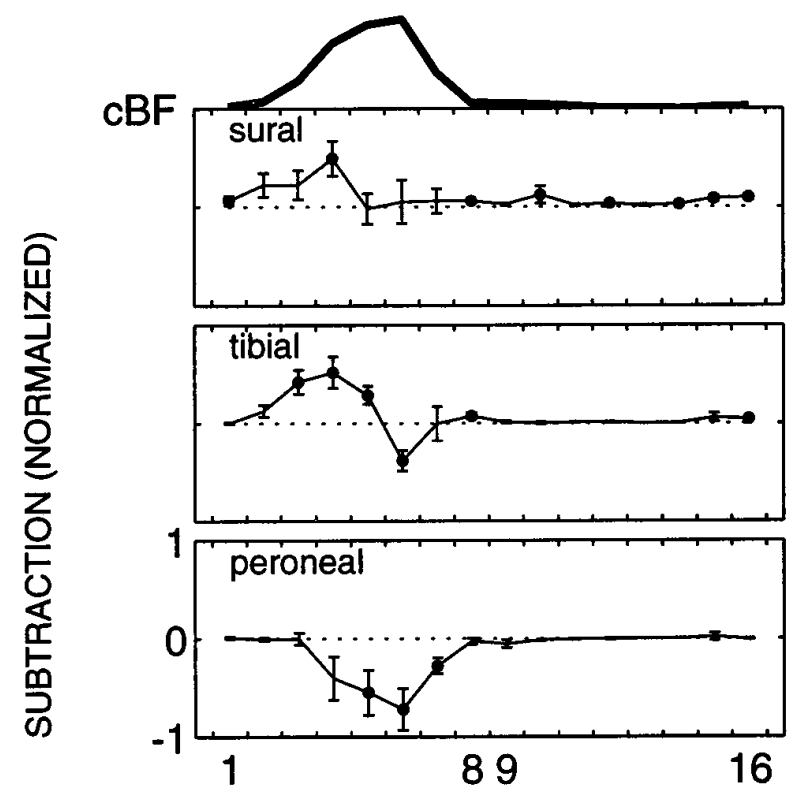

PHASE IN STEP CYCLE

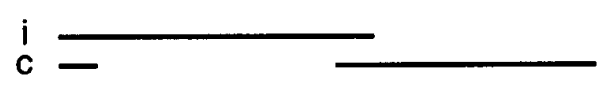

Figure 6. Contralateral biceps femoris $(c B F)$ of subject 2. The same format is used as in Figure 2.

In other periods of the step cycle the iTA responses could be different for the three nerves. During most of the stance phase both sural and peroneal nerve stimulation generally did not evoke responses in iTA, whereas tibial nerve stimulation evoked small facilitatory responses (Figs. 2, 3). For the three nerves the iTA responses generally changed around the stance-to-swing period. For example, the small or absent sural nerve responses during early to mid stance changed in most subjects to facilitations (Fig. 3) and, in one subject, to suppressions (Fig. 2) during late stance to early swing.

The ipsilateral ankle angles were measured to check whether the stimulation also evoked mechanical responses. Usually there were no large effects observed (see also Duysens et al., 1992). However, some subjects showed small but clear effects that were $\sim 5^{\circ}$ at maximum. These kinesiological responses occurred predominantly during the swing phase. Results are given in Figure 4 for the early swing (mean of phases 10-11), mid-swing (phases 12-14), and late swing (phases 15 and 16) periods. The sural nerve evoked dorsiflexion from early swing to mid-swing (resulting from the predominantly facilitatory responses in iTA; see Fig. 3). In contrast, during the same period both the tibial and peroneal nerves evoked predominantly plantar flexion movements (Fig. 4). Hence, during early swing to mid-swing the evoked kinesiological effect depended on the location of the stimulus. All three nerves evoked plantar flexion during late swing (Fig. 4), consistent with the suppressive responses in iTA during this period (Fig. 3).

\section{Contralateral responses}

For all three nerves EMG responses were elicited contralateral to the side of stimulation, as well. Population averages are given in Figure 5. The responses were, on average, smaller as compared with the ipsilateral responses. Nevertheless, clear modulation patterns were observed, which were usually different from the ipsilateral modulation patterns in the same muscles.

In contrast to iBF and iST, $\mathrm{cBF}$ and cST generally showed a similar response synergy across the step cycle for all three nerves (Fig. 5). An overall facilitatory pattern was found, with the largest responses occurring during contralateral mid to late stance (phases 12-16). This was in contrast with the background activity, because the latter was largest during the contralateral swing phase (phases 6-9). Hence, there is an uncoupling of reflex and background magnitude in the contralateral leg.

During contralateral stance the responses in $\mathrm{cBF}$ and $\mathrm{cST}$ were generally similar for the three nerves (Fig. 5). However, during contralateral swing the response modulation tended to be different. Peroneal stimulation, on average, caused suppressions in $\mathrm{cBF}$ and $\mathrm{cST}$, whereas facilitations clearly prevailed for the sural nerve. A mixture of facilitations and suppressions was observed for the tibial nerve (Fig. 5). In four subjects statistically significant facilitations to sural nerve stimulation during this period were accompanied by statistically significant suppressions to peroneal nerve stimulation. An example is shown in Figure 6.

cRF generally showed responses during the periods in which the muscle was spontaneously active (stance-to-swing and swingto-stance transitions). During the swing-to-stance transition (phases 7-10), stimulation of the sural and tibial nerves evoked facilitatory responses that were usually larger than the responses to peroneal nerve stimulation during this period (Fig. 5).

Responses in cTA were small for all three nerves. Most commonly, small facilitatory responses were evoked during contralateral stance, and suppressions were evoked during contralateral swing (Fig. 5).

\section{DISCUSSION}

The major new finding from this study is that non-nociceptive stimulation of the sural, posterior tibial, and superficial peroneal nerves each evokes distinct reflex responses, indicating the presence of location-specific information from the skin of the foot in cutaneous reflexes during human walking. The nerve-specific phase-dependent reflex modulation patterns point to the dynamic control of this information during the course of a step cycle.

Reflex responses from different nerves during walking

After non-nociceptive stimulation all three nerves under investigation exhibited reflex responses with a latency of $\sim 80 \mathrm{msec}$ and a duration of $\sim 30 \mathrm{msec}$ for all muscles, both ipsilateral and contralateral to the side of stimulation. For all three nerves the magnitude of these responses varied as a function of the phase in the step cycle in a way that could not be related to variations in stimulus intensity (see Materials and Methods) or background activity levels. The latency, duration, and the phase-dependent reflex modulation patterns for the sural (all muscles) and tibial (iTA) nerves correspond to (and are in good agreement with) the "middle latency" or "P2" responses in related literature on the reflex responses for individual nerves (Duysens et al., 1990, 1991, 1996; Yang and Stein, 1990; De Serres et al., 1995; Tax et al., 1995). These data are now for the first time complemented with results on superficial peroneal stimulation. 
The reflex modulation was not closely related to the level of background EMG activity in any of the nerve/muscle combinations. Hence, the approximately linear relation of reflex responses with the magnitude of the background activity (and thus presumably with the motoneuronal excitability) in static conditions (Matthews, 1986) does not apply during locomotion. Additional mechanisms at a premotoneuronal level therefore must participate in the phase-dependent control of the reflex responses for the three nerves, consistent with the literature (Forssberg, 1979; Duysens and Loeb, 1980; Kanda and Sato, 1983; Abraham et al., 1985; Drew and Rossignol, 1987; Duysens et al., 1990, 1991; Yang and Stein, 1990; Pratt et al., 1991; LaBella et al., 1992; De Serres et al., 1995; Tax et al., 1995; Degtyarenko et al., 1996).

The reflex responses depended on the stimulated nerve, as would be expected if the location of the stimulus is important in the outcome of the observed responses. This may be a general phenomenon, because location-specific reflex effects have been observed during locomotion of the cat (Abraham et al., 1985; Moschovakis et al., 1991; Pratt et al., 1991; LaBella et al., 1992; Degtyarenko et al., 1996) and even during locomotion of invertebrates with a relatively simple nervous system such as the locust (Laurent and Hustert, 1988). Nevertheless, this is the first study to describe nerve-specific reflex effects during human locomotion. The results indicate the presence of specializations in the reflex pathways of the various nerves, which can be used to provide spatially tuned sensory information from the skin of the foot during human locomotion, as will be discussed below.

\section{Control of location-specific information in cutaneous reflexes during walking}

The nerve-specific responses in single muscles indicate a segregation of the reflex pathways that are used during human walking. Still, reflex effects from different nerves or skin areas have been found in the same motoneurons in the cat (Hagbarth, 1952; Engberg, 1964; Dum and Kennedy, 1980; Loeb et al., 1987; LaBella et al., 1989; LaBella and McCrea, 1990; Moschovakis et al., 1991; Degtyarenko et al., 1996), in the rat (Woolf and Swett, 1984; Cook and Woolf, 1985), and in the locust (Siegler and Burrows, 1986; Laurent and Hustert, 1988), indicating convergence somewhere on the reflex pathways. This also would apply to man, because common responses were observed in the same motor units of human arm muscles (Garnett and Stephens, 1980) and human leg muscles (Aniss et al., 1988, 1992) under stationary conditions. For low-threshold cutaneous afferents the dominant site of convergence (or "final common pathway") seems to be the motoneuronal level, because in the cat the largest degree of interneuronal convergence existed for nerves that have overlapping receptive fields, whereas such convergence of nerves with separate receptive fields was relatively restricted (LaBella and McCrea, 1990). This is not unlike what has been observed for high-threshold nociceptive pathways. In the rat, functional withdrawal reflexes recently were observed to have a very distinct local sign (Schouenborg and Kalliomäki, 1990; Schouenborg et al., 1992), leading to a new hypothesis that nociceptive withdrawal reflexes are organized by "functionally separate reflex pathways to separate muscles or small groups of synergistic muscles" (Schouenborg et al., 1994).

Hence, if we assume the existence of reflex pathways from the various cutaneous nerves to the same motoneurons, the observed nerve specificity must arise primarily at a premotoneuronal level. The reflex pathways to the motoneurons can be different in the overall strength of the excitatory and inhibitory connections. The large iBF responses after sural nerve stimulation in the stance phase then could be explained by strong reflex connections (Hugon, 1973), whereas the smaller iBF responses for the peroneal nerve in the same period then could be explained by weaker reflex connections. However, the nerve specificity of the responses depended on the phase of the step cycle, because the iBF responses were of the same order of magnitude for these two nerves during swing. In some phases the peroneal responses even tended to be larger. Such overlapping response amplitudes cannot be explained solely by differences in the strength of reflex connections for the various nerves. Rather, these results indicate a differential and, thus, separate control over the reflex pathways of the three nerves at a premotoneuronal level. In that case, for example, the excitatory reflex pathways of the sural nerve to $\mathrm{iBF}$ would be (partially) "open" during both stance and swing, whereas the peroneal pathways would be "open" during swing and "closed" during stance. In other words, the results point to a dynamic control of location-specific information during the course of a step cycle.

Still, some strikingly similar reflex effects for the three nerves were observed as well, especially in the contralateral muscles. In the ipsilateral muscles these could occur in particular during the stance-to-swing and the swing-to-stance periods. For example, all three nerves evoked suppressions in iTA during end swing. With the clear indications of the existence of segregated parts in the reflex pathways of the three nerves, any such observations could be explained by a synchronized premotoneuronal control of the reflex pathways of the three nerves to a given set of motoneurons. However, such observations also could be explained by a convergence of the reflex pathways onto common interneurons. In that case, the presently observed responses would be mediated in part by common pathways and in part by private pathways (Hagbarth, 1952; see also LaBella and McCrea, 1990) that are the points of differential modulation at specific phases in the step cycle.

The results also show that reflex synergies can be specialized according to the stimulated nerve. It was already known that sural nerve stimulation elicits a differential response pattern of predominant facilitatory responses in $\mathrm{iBF}$ and substantial smaller or even suppressive responses in iST (Tax et al., 1995; Duysens et al., 1996) (see also Figs. 2, 3). Because the coordinated action could contribute to foot exorotation, it was hypothesized that this reflex synergy could be related exclusively to the innervation area of the sural nerve during human locomotion (Tax et al., 1995). The present data support this hypothesis.

\section{Functional implications}

On the basis of the sural nerve responses during human running, it was suggested that "tactile cutaneous feedback may be used to move the perturbed leg away from the stimulus, with the general constraint of preserving both the cadence and the balance at all times during the step cycle" (Tax et al., 1995). The presently observed sural nerve responses are completely comparable with those obtained during running.

During everyday walking the dorsum of the foot, innervated by the peroneal nerve, is most likely to encounter an external stimulus or obstacle during the swing phase. In cats such a perturbation evokes a "stumbling corrective reaction" (Forssberg, 1979; Drew and Rossignol, 1987; Buford and Smith, 1993), which was either reduced or abolished by anesthetizing the corresponding skin area (Drew and Rossignol, 1987). In the present study, the suppressive responses in most subjects in iTA to peroneal stimulation during early swing would induce ankle plantar flexion (see 
Fig. 4), while at the same time the large facilitations in both iBF and iST in combination with the low responses in iRF could induce hip extension and/or knee flexion. The foot would be cleared from the obstacle to prepare the leg to step over it, thus enabling continuation of an ongoing walking pattern. On the other hand, during end swing the $\mathrm{iBF}$, iST, and iRF response synergy is comparable to that of the normal ongoing activity. Hence, it would induce an additional deceleration of the leg in preparation for stance (Inman et al., 1981). In combination with the iTA suppressions the functional role could be twofold. First, the response synergy would be directed at an early placing, creating early stability. Second, the leg would move away from the stimulus. Note that these peroneal response synergies could correspond to the functional responses after actual obstruction of the forward swinging leg during both early ("elevating strategy": Eng et al., 1994; Schillings et al., 1996) and late swing ("lowering strategy": Eng et al., 1994).

The plantar surface of the foot, innervated by the posterior tibial nerve, could provide (phasic) information about the ground surface, because it will be in contact with the environment predominantly during the stance phase. The facilitations in iTA to tibial stimulation during middle and late stance would induce a reduction of ankle plantar flexion (as occasionally observed by Duysens et al., 1990), perhaps to reduce impact with the perturbation. The facilitations in $\mathrm{iRF}$ in combination with the small or absent responses in $\mathrm{BBF}$ and iST could stabilize the standing leg by an additional knee extension moment. During late swing the iBF and iST suppressions in combination with the iRF facilitations would reduce deceleration of the leg, which would increase step length. In combination with the iTA suppressions (plantar flexion) the reflex synergy would reduce contact with stimulus and still prepare for a safe footfall.

In summary, tactile cutaneous feedback from the foot contains location-specific information that seems to be incorporated in a functional way in reflexes during human walking. Balance and cadence seem to have priority over a moving away from the stimulus, consistent with the above-mentioned hypothesis proposed by Tax et al. (1995). So that the functional role of tactile cutaneous feedback can be understood better, however, the kinesiological changes resulting from the reflex responses should be studied more in detail because these were at best only small (see also Duysens et al., 1992). Still, the present results already indicate that location-specific information from the skin of the foot is sufficiently important to warrant the observed differential control of this information during human walking.

\section{REFERENCES}

Abraham LD, Marks WB, Loeb GE (1985) The distal hindlimb musculature of the cat. Cutaneous reflexes during locomotion. Exp Brain Res 58:594-603.

Aniss AM, Gandevia SC, Burke D (1988) Reflex changes in muscle spindle discharge during a voluntary contraction. J Neurophysiol 59:908-921.

Aniss AM, Gandevia SC, Burke D (1992) Reflex responses in active muscles elicited by stimulation of low-threshold afferents from the human foot. J Neurophysiol 67:1375-1384.

Baker SN, Lemon RN (1995) Non-linear summation of responses in averages of rectified EMG. J Neurosci Methods 59:175-181.

Buford JA, Smith JL (1993) Adaptive control for backward quadrupedal walking. III. Stumbling corrective reactions and cutaneous reflex sensitivity. J Neurophysiol 70:1102-1114.

Cook AJ, Woolf CJ (1985) Cutaneous receptive field and morphological properties of hamstring flexor-motoneurones in the rat. J Physiol (Lond) 364:249-263.

Degtyarenko AM, Simon ES, Burke RE (1996) Differential modulation of disynaptic cutaneous inhibition and excitation in ankle flexor motoneurons during fictive locomotion. J Neurophysiol 76:2972-2985.

De Serres SJ, Yang JF, Patrick SK (1995) Mechanism for reflex reversal during walking in human tibialis anterior muscle revealed by single motor unit recording. J Physiol (Lond) 488:249-258.

Drew T, Rossignol S (1987) A kinematic and electromyographic study of cutaneous reflexes evoked from the forelimb of unrestrained walking cats. J Neurophysiol 57:1160-1184.

Dum RP, Kennedy TT (1980) Synaptic organization of defined motorunit types in cat tibialis anterior. J Neurophysiol 43:1631-1644.

Duysens J, Loeb GE (1980) Modulation of ipsi- and contralateral reflex responses in unrestrained walking cats. J Neurophysiol 44:1024-1037.

Duysens J, Stein RB (1978) Reflexes induced by nerve stimulation in walking cats with implanted cuff electrodes. Exp Brain Res 32:213-224.

Duysens J, Trippel M, Horstmann M, Dietz V (1990) Gating and reversal of reflexes in ankle muscles during human walking. Exp Brain Res 82:351-358.

Duysens J, Tax AAM, Doelen B, Van der Trippel M, Dietz V (1991) Selective activation of human soleus or gastrocnemius in reflex responses during walking and running. Exp Brain Res 87:193-204.

Duysens J, Tax AAM, Trippel M, Dietz V (1992) Phase-dependent reversal of reflexly induced movements during human gait. Exp Brain Res 90:404-414.

Duysens J, Tax AAM, Nawijn S, Berger W, Prokop T, Altenmüller E (1995) Gating of sensation and evoked potentials following foot stimulation during human gait. Exp Brain Res 105:423-431.

Duysens J, Tax AAM, Murrer L, Dietz V (1996) Backward and forward walking use different patterns of phase-dependent modulation of cutaneous reflexes in humans. J Neurophysiol 76:301-310.

Eng JJ, Winter DA, Patla AE (1994) Strategies for recovery from a trip in early and late swing during human walking. Exp Brain Res 102:339-349.

Engberg I (1964) Reflexes to foot muscles in the cat. Acta Physiol Scand 62[Suppl 235]:1-64.

Forssberg H (1979) Stumbling corrective reaction: a phase-dependent compensatory reaction during locomotion. J Neurophysiol 42:936-953.

Garnett R, Stephens JA (1980) The reflex responses of single motor units in human first dorsal interosseous muscle following cutaneous afferent stimulation. J Physiol (Lond) 303:351-364.

Hagbarth KE (1952) Excitatory and inhibitory skin areas for flexion and extensor motoneurons. Acta Physiol Scand 26[Suppl 94]:1-58.

Hagbarth KE (1960) Spinal withdrawal reflexes in the human lower limbs. J Neurol Neurosurg Psychiatry 23:222-227.

Hongo T, Kudo N, Oguni E, Yoshida K (1990) Spatial patterns of reflex responses evoked by pressure stimulation of the foot pads in cats. J Physiol (Lond) 420:471-487.

Hugon M (1973) Exteroceptive reflexes to stimulation of the sural nerve in normal man. In: New developments in electromyography and clinical neurophysiology, Vol 3 (Desmedt JE, ed), pp 713-729. Basel: Karger.

Inman VT, Ralston HJ, Todd F (1981) Human walking. Baltimore: Williams \& Wilkins.

Kanda K, Sato H (1983) Reflex responses of human thigh muscles to non-noxious sural stimulation during stepping. Brain Res 288:378-380.

Kugelberg E, Eklund K, Grimby L (1960) An electromyographic study of the nociceptive reflexes of the lower limb. Mechanism of the plantar responses. Brain 53:394-410.

LaBella LA, McCrea DA (1990) Evidence for restricted central convergence of cutaneous afferents on an excitatory reflex pathway to medial gastrocnemius motoneurons. J Neurophysiol 64:403-412.

LaBella LA, Kehler JP, McCrea DA (1989) A differential synaptic input to the motor nuclei of triceps surae from the caudal and lateral cutaneous sural nerves. J Neurophysiol 61:291-301.

LaBella LA, Niechaj A, Rossignol S (1992) Low-threshold, short-latency cutaneous reflexes during fictive locomotion in the "semi-chronic" spinal cat. Exp Brain Res 91:236-248.

Laurent G, Hustert R (1988) Motor neuronal receptive fields delimit patterns of motor activity during locomotion of the locust. J Neurosci 8:4349-4366.

Leahy JC, Durkovic RG (1991) Differential synaptic effects on physiological flexor hindlimb motoneurons from cutaneous nerve inputs in spinal cat. J Neurophysiol 66:460-472.

Loeb GE, Marks WB, Hoffer JA (1987) Cat hindlimb motoneurons during locomotion. IV. Participation in cutaneous reflexes. J Neurophysiol 57:563-573.

Matthews PBC (1986) Observations on the automatic compensation of 
reflex gain on varying the pre-existing level of motor discharge in man. J Physiol (Lond) 374:73-90.

Meinck HM, Piesiur-Strehlow B, Koehler W (1981) Some principles of flexor reflex generation in human leg muscles. Electroencephalogr Clin Neurophysiol 52:140-150.

Moschovakis AK, Sholomenko GN, Burke RE (1991) Differential control of short latency cutaneous excitation in cat FDL motoneurons during fictive locomotion. Exp Brain Res 83:489-501.

Pratt CA, Chanaud CM, Loeb GE (1991) Functionally complex muscles of the cat hindlimb. IV. Intramuscular distribution of movement command signals and cutaneous reflexes in broad, bifunctional thigh muscles. Exp Brain Res 85:281-299.

Rossignol S, Lund JP, Drew T (1988) The role of sensory inputs in regulating patterns of rhythmical movements in higher vertebrates. A comparison between locomotion, respiration, mastication. In: Neural control of rhythmic movements in vertebrates (Cohen A, Rossignol S, Grillner S, eds), pp 210-283. New York: Wiley.

Schillings AM, Van Wezel BMH, Duysens J (1996) Mechanically induced stumbling during human treadmill walking. J Neurosci Methods 67:11-17.

Schouenborg J, Kalliomäki J (1990) Functional organization of the nociceptive withdrawal reflexes. I. Activation of hindlimb muscles in the rat. Exp Brain Res 83:67-78.
Schouenborg J, Holmberg H, Weng HR (1992) Functional organization of the nociceptive withdrawal reflexes. II. Changes of excitability and receptive fields after spinalization in the rat. Exp Brain Res 90:469-478.

Schouenborg J, Weng HR, Holmberg H (1994) Modular organization of spinal nociceptive reflexes: a new hypothesis. News Physiol Sci 9:261-265.

Sherrington CS (1910) Flexion-reflex of the limb, crossed extension reflex, reflex stepping, and standing. J Physiol (Lond) 40:28-121.

Siegler MV, Burrows M (1986) Receptive fields of motor neurons underlying local tactile reflexes in the locust. J Neurosci 6:507-513.

Tax AAM, Van Wezel BMH, Dietz V (1995) Bipedal reflex coordination to tactile stimulation of the sural nerve during human running. J Neurophysiol 73:1947-1964.

Van Wezel BMH, Ottenhoff FAM, Duysens J (1994) Reflex responses to low-intensity stimulation of the sural, tibial, and peroneal nerves during human walking. Soc Neurosci Abstr 20:1582.

Woolf CJ, Swett JE (1984) The cutaneous contribution to the hamstring flexor reflex in the rat: an electrophysiological and anatomical study. Brain Res 303:299-312.

Yang JF, Stein RB (1990) Phase-dependent reflex reversal in human leg muscles during walking. J Neurophysiol 63:1109-1117. 